\title{
Linfoma de Hodgkin: Análise de desfechos em óbito no Brasil, na região Norte e no
}

\section{Amapá em uma década}

\author{
Hodgkin's lymphoma: Analysis of outcomes dying in Brazil, in the North region and Amapá in a \\ decade \\ Linfoma de Hodgkin: Análisis de resultados morir en Brasil, en la región Norte y Amapá en una \\ década
}

\author{
Lucas Almeida Ribeiro \\ ORCID: https://orcid.org/0000-0003-4031-9027 \\ Universidade Federal do Amapá, Brasil \\ E-mail: gplucas@outlook.com \\ Antônio Bessa de Castro Filho \\ ORCID: https://orcid.org/0000-0002-8198-3111 \\ Universidade Federal do Amapá, Brasil \\ E-mail: antoniobessa96@yahoo.com \\ João Ferreira Gonçalves Neto \\ ORCID: https://orcid.org/0000-0002-1726-4083 \\ Universidade Federal do Amapá, Brasil \\ E-mail: joaofgneto@gmail.com \\ Jorge Lucas Alves Santana \\ ORCID: https://orcid.org/0000-0001-7246-9535 \\ Universidade Federal do Rio Grande do Norte, Brasil \\ E-mail: jorgelucas.as@hotmail.com \\ Thiago Lima Peixoto Costa \\ ORCID: https://orcid.org/0000-0003-3638-8847 \\ Universidade Federal do Amapá, Brasil \\ E-mail: thiagolimapeixoto@gmail.com \\ Maria Helena Mendonça de Araújo \\ ORCID: https://orcid.org/0000-0002-7742-144X \\ Universidade Federal do Amapá, Brasil \\ E-mail: ma.helenam@hotmail.com
}

\begin{abstract}
Resumo
O linfoma de Hodgkin é uma doença maligna caracterizada por uma massa contendo células linfoides e reacionais provenientes de uma expansão clonal dos sistemas hematopoiético e reticuloendotelial. Sua incidência possui um pico bimodal, sendo os jovens adultos e idosos os grupos etários mais atingidos. Este artigo analisa o desfecho em óbitos da doença no Brasil, na região norte e no estado do Amapá no período de 10 anos. O estudo apresentado é do tipo epidemiológico descritivo, documental e retrospectivo, os dados foram obtidos por meio do atlas online de mortalidade por câncer do INCA. No Brasil observou-se crescimento nos primeiros anos e oscilação do número no restante em todas as regiões, a faixa etária com maior taxa são os adultos e o sexo masculino predomina sobre o feminino. A região norte segue o padrão brasileiro, com destaque aos estados do Pará e do Amazonas que tem maior mortalidade. O Amapá fica em quinto lugar na região Norte, concentrando o maior número em sua capital, Macapá. A região sudeste do país concentra maior número de casos, fatores como maior densidade populacional, maior número de infectados pelo HIV e a concentração de centros especializados de tratamento oncológico corroboram para isso. O número de mortes no país concorda com o formato de incidência bimodal que a doença apresenta. Ações de informação, notificação, prevenção e tratamento da doença devem ser mais encorajadas no Brasil, pois o diagnóstico precoce permite melhor prognóstico e diminuição no número de óbitos.Incluir o resumo.
\end{abstract}

Palavras-chave: Linfoma de Hodgkin; Registros de óbito; Epidemiologia.

\begin{abstract}
Hodgkin's lymphoma is a malignant disease, characterized by a mass wich contains lymphoid and reactive cells resulting from a clonal expansion of the hematopoietic and reticuloendothelial systems. Its incidence has a bimodal peak, with young adults and the elderly being the most affected age groups. This article seeks to analyze the outcome of deaths from this disease in Brazil, in the Northern region and in the state of Amapá, over a period of 10 years. The study presented is epidemiologically descriptive, documentary and retrospective. The data were obtained through the
\end{abstract}


online atlas of cancer mortality from INCA. In Brazil, growth was observed in the first years and the numbers fluctuated in the coming years. In all regions, the age group with the highest index is adults and man predominate over women. The northern region follows the brazilian pattern, with emphasis on the states of Pará and Amazonas, which have the highest mortality rates. Amapá occupies the fifth place in the Northen region, concentrating the largest number in its capital, Macapá. The southeastern region of the country concentrates the largest number of cases, and factors such as a greater population density, greater number of people infected with HIV and the concentration of centers specialized in cancer treatment corroborate this. The number of deaths in the country is in accordance with the bimodal incidence format of the disease. Information, notification, prevention and treatment of the disease should be promoted more in Brazil, since an early diagnosis allows a better prognosis and a reduction in the number of deaths.

Keywords: Hodgkin's lymphoma; Death record; Epidemiology.

\section{Resumen}

El linfoma de Hodgkin es una enfermedad maligna caracterizada por una masa que contiene células linfoides y reactivas como resultado de una expansión clonal del sistemas hematopoyético y reticuloendotelial. Su incidencia tiene un pico bimodal, siendo los adultos jóvenes y los ancianos los grupos de edad más afectados. Este artículo analiza el resultado de las muertes por la enfermedad en Brasil, en la región norte y en el estado de Amapá durante un período de 10 años. El estudio que se presenta es descriptivo, documental y epidemiológico retrospectivo, los datos fueron obtenidos a través del atlas online de mortalidad por cáncer del INCA. Se observó crecimiento en los primeros años y el número fluctuó en el los demás, así como en Brasil, en todas las regiones, el grupo de edad con mayor índices es el adulto y predominan los hombres sobre las mujeres. La región norte sigue el patrón brasileño, con énfasis en los estados de Pará y Amazonas, que tienen las tasas de mortalidad más altas. Amapá ocupa el quinto lugar en el norte, concentrando el mayor número en su capital, Macapá. La región sureste del país concentra la mayor cantidad de casos, factores como mayor densidad poblacional, mayor número de personas infectadas por el VIH y la concentración de centros especializados en el tratamiento del cáncer lo corroboran. El número de muertes en el país concuerda con el formato de incidencia bimodal que presenta la enfermedad. Se debe promover más la información, notificación, prevención y tratamiento de la enfermedad en Brasil, ya que el diagnóstico precoz permite un mejor pronóstico y la reducción del número de muertes.

Palabras clave: Linfoma de Hodgkin; Acta de defunción; Epidemiología.

\section{Introdução}

Os linfomas são um grupo de tumores provenientes do sistema hematopoiético e têm como característica fundamental a extensa proliferação de células linfoides (linfócitos) bem como de seus precursores. Podem ser classificados em dois grandes tipos, o linfoma de Hodgkin (LH) e o linfoma não-Hodgkin (LNH), e possuem subtipos dependendo da sua caracterização pelo tipo de célula linfoide envolvida (Brasil, 2020; Storck, Brandstetter, Keller, \& Knopf, 2019).

A neoplasia conhecida por linfoma de Hodgkin decorre pelo aparecimento das células de Reed-Sternberg (RS), que são linfócitos do tipo B com derivação maligna por proliferação clonal, e células de Hodgkin. As células RS são minoria no tecido tumoral encontrado, sendo cercadas de outras células reacionais e não cancerígenas, como linfócitos T, neutrófilos, eosinófilos, fibroblastos, mastócitos, macrófagos e células plasmáticas. Possuem como características histológicas o tamanho largo, a presença de dois lobos com diferentes nucléolos, com uma aparência de "olhos de coruja", enquanto que as células de Hodgkin possuem nucléolos aumentados (Brasil, 2020; Fields \& Wrench, 2017; Santos \& Lima, 2017).

No Brasil, em todos os estados, a estimativa do número de casos da doença para o ano de 2020, para cada 100 mil habitantes é de 1.590 nos homens e 1.050 nas mulheres. Demonstrando uma expectativa de ser a $14^{\mathrm{a}}$ mais incidente neoplasia no sexo masculino e a $17^{\mathrm{a}}$ doença maligna com maior incidência no sexo feminino, excetuando-se o câncer de pele não melanoma (Instituto Nacional do Câncer [INCA], 2020b).

De acordo com a Organização Mundial da Saúde (OMS), o LH pode ser subdividido em duas classificações, o LH clássico e o LH de predomínio linfocitário nodular, sendo o primeiro responsável por cerca de $90 \%$ dos casos. Este ainda pode receber uma subclassificação em quatro grupos: esclerose nodular; celularidade mista; ricos em linfócitos e esgotados de linfócitos, respectivamente em ordem decrescente de incidência (Brasil, 2020).

A incidência do LH é maior em adultos jovens e uma associação com o vírus Epstein-Barr (EBV) pode ser encontrada em sua patogênese. Por outro lado, um segundo pico da doença ocorre em idosos, pois estes possuem sua imunocompetência 
debilitada devido à senescência fisiológica, dividindo um processo patológico comum a outros linfomas e doenças malignas. Contudo, o risco de desenvolver a doença após contaminação por EBV é muito pequeno posto que o genoma viral é encontrado em uma pequena amostra de subtipo do LH (Brasil, 2020; Gobbi, Ferreri, Ponzoni, \& Levis, 2013).

Hjalgrim et al. (2003) concluem que pessoas com mononucleose infecciosa tem risco elevado de desenvolver HL associada ao EBV, principalmente em adultos. Além disso, Gobbi et al. (2013) afirmam que pessoas com exposição sistêmica a sangue ou produtos sanguíneos, como usuários de drogas intravenosas e portadores de hemofilia, doença de coagulação sanguínea, possuem alto risco de apresentar LH e são, presumidamente, não portadoras do vírus da imunodeficiência humana (HIV). Deve-se considerar neste contexto também pessoas que são desconhecedoras de seu estado sorológico para o HIV, ou seja, são pessoas vivendo com o HIV, porém não diagnosticadas e deve ser feita busca ativa destes, diante do diagnóstico de LH dado que a presença do vírus possui relação bem estabelecida de substancial risco elevado a desenvolver LH.

Ainda acerca da incidência da doença, um dado interessante entre países subdesenvolvidos ou em desenvolvimento e desenvolvidos economicamente é que o LH é mais incidente em jovens adultos nos desenvolvidos, enquanto que o número de casos de crianças com menos de quinze anos apresentam uma incidência mais acentuada nos outros países (Gobbi et al., 2013).

A doença se apresenta com linfadenopatia indolor nas regiões cervical e/ou supraclavicular, febre, perda não intencional de mais de $10 \%$ do peso corporal habitual no período de seis meses e sudorese noturna, esses três últimos são chamados de sintomas B ou constitucionais e possuem relevância negativa quanto ao prognóstico. Tosse, dispneia, fadiga, odino e disfagia são sintomas ligados ao aparecimento de massa em regiões do mediastino e cervical (Brasil, 2020; Santos \& Lima, 2017; Storck et al, 2019).

O estadiamento do LH pode ser feito por dois sistemas de classificação com diferentes metodologias e são utilizados a depender do serviço e seus recursos disponíveis. O primeiro é a classificação de Ann Arbor, no qual os três primeiros estádios indicam o grau de desenvolvimento linfonodal, enquanto que o quarto classifica quanto ao acometimento extra-linfonodal, ou seja, disseminado. Ademais, esta classificação considera a ausência ou a presença dos sintomas constitucionais, identificados em A ou B, respectivamente. Outrossim, a classificação de Lugano que utiliza como método a tomografia computadorizada por emissão de pósitrons (PET-TC) no momento do diagnóstico e na avaliação das respostas terapêuticas. Este possui uma sensibilidade maior à percepção de massas, linfonodos e órgãos acometidos, principalmente fígado e baço (Brasil, 2020; Carbone, Kaplan, Musshoff, Smithers, \& Tubiana, 1971; Cheson et al., 2014).

Pacientes submetidos ao tratamento, a depender do estágio ao diagnóstico e fatores de risco envolvidos, podem entrar em remissão da doença em cinco anos. Apesar de protocolos eficazes e com bons prognósticos, até alcance de cura em alguns pacientes, outros são refratários ou apresentam recorrência do linfoma, cerca de $10 \%$ e 20 a $25 \%$, respectivamente (Duarte et al., 2016; Küppers, Engert, \& Hansmann, 2012).

A importância de desenhar o prognóstico do paciente levou ao desenvolvimento de diversos recursos a fim de mensurar a chance de sobrevida bem como instituir o tratamento apropriado a cada paciente, ao passo que isto corrobora a uma análise retrospectiva pós-terapêutica (Brasil, 2020).

Este artigo tem por objetivo analisar os desfechos em óbito no Brasil, na região norte do país e no estado do Amapá e direcionar a comparação entre as características epidemiológicas entre as localidades estudadas com a bibliografia vigente.

\section{Metodologia}

O estudo é do tipo epidemiológico descritivo, documental e retrospectivo. Os dados da pesquisa foram coletados por meio do sítio do Departamento de informática do SUS (DATASUS). A partir do DATASUS foi possível acessar o atlas online de mortalidade por câncer do Instituto Nacional de Câncer [INCA] (2020a). Esses dados foram acessados no dia 11 de Novembro de 2020. 
Para a obtenção dos dados acerca dos desfechos em óbito do linfoma de Hodgkin seguiu-se a seguinte sequência: no sítio do DATASUS clicou-se em Informações à saúde (TABNET). Após o redirecionamento para a página do TABNET, selecionaram-se as opções "Estatísticas Vitais"; "Câncer (sítio do INCA)”; "Atlas de Mortalidade por Câncer”. Dessa maneira, no sítio do Atlas On-line de Mortalidade, escolheu-se a opção "Taxas de mortalidade por câncer, brutas e ajustadas por idade pelas populações mundial e brasileira, por 100.000, segundo sexo, faixa etária, localidade e por período selecionado" e configurou-se o período: de 2009 a 2018; as regiões: Brasil, Centro-oeste, Nordeste, Norte, Sudeste e Sul; apenas para região Norte foi selecionado o subtópico estado: Acre, Amapá, Amazonas, Pará, Rondônia, Roraima e Tocantins; apenas para o estado do Amapá, foi selecionado o subtópico cidades: Amapá, Macapá, Porto Grande e Santana; população brasileira: Brasil 2010; a topografia: tipo de câncer - "C81- DOENCA DE HODGKIN”.

As referências bibliográficas foram pesquisadas através de artigos científicos presentes na biblioteca Scientific Electronic Library Online (SciELO), no Portal de periódicos da Coordenação de aperfeiçoamento de pessoal de nível superior (CAPES), na base de dados Literatura Latino-Americana em Ciências da Saúde (Lilacs) e em cartilhas e sítios oficiais do Ministério da saúde (MS). As tabelas foram editadas utilizando o programa Word software Microsoft Office 2010. Na organização da faixa etária, os números foram compilados seguindo os critérios adotados pelo Instituto Brasileiro de Geografia e Estatística: "Jovens", de 0 a 19 anos; "Adultos”, de 20 a 59 anos e "Idosos”, para maiores de 60 anos.

Esse estudo está de acordo com a Resolução número 466 de 2012 do Conselho Nacional de Saúde (CNS), uma vez que os dados utilizados são secundários, isentando o enfoque em pessoas ou instituições e não permitindo a identificação individual.

\section{Resultados}

Durante a década analisada - 2009 a 2018 - foram notificados 5.319 óbitos causados pelo LH no Brasil. O Sudeste foi a região brasileira com maior número de notificações, sendo responsável por $49,40 \%$ ( $\mathrm{n}=2628)$ dos casos, enquanto a região Norte registrou o menor número, apenas 5,35\% ( $\mathrm{n}=285)$. Entre os estados que compõe o Norte brasileiro, o Pará se destaca com o maior número de notificações, 43,15\% ( $\mathrm{n}=123)$, enquanto que o Acre aparece com o menor número, 3,15\% ( $\mathrm{n}=9$ ). $\mathrm{O}$ estado do Amapá registrou 15 mortes pelo LH, sendo 93,33\% ( $\mathrm{n}=14)$ na capital, Macapá. Em relação ao sexo, dos pacientes com desfecho de óbito os homens foram maioria, sendo no Brasil 56,29\% ( $\mathrm{n}=2994)$, na região Norte 67,01\% ( $\mathrm{n}=191)$ e no estado do Amapá 56,25\% ( $\mathrm{n}=9$ ). A faixa etária de mortes predominante, nos cenários estudados, foi adulta, seguida pelos idosos e jovens. Os dados são detalhados nas tabelas a seguir.

Na Tabela 1, observam-se pequenas variações no número de notificações por mortes causadas pelo LH ao longo da década. Os casos cresceram entre 2009 a 2012, havendo uma discreta queda no biênio de 2013 e 2014, e terminaram a década com oscilações de crescimentos e quedas entre 2015 a 2018.

Tabela 1: Número de óbitos pelo Linfoma de Hodgkin no Brasil, por região entre 2009 e 2018.

\begin{tabular}{cccccccccccc}
\hline & $\mathbf{2 0 0 9}$ & $\mathbf{2 0 1 0}$ & $\mathbf{2 0 1 1}$ & $\mathbf{2 0 1 2}$ & $\mathbf{2 0 1 3}$ & $\mathbf{2 0 1 4}$ & $\mathbf{2 0 1 5}$ & $\mathbf{2 0 1 6}$ & $\mathbf{2 0 1 7}$ & $\mathbf{2 0 1 8}$ & TOTAL \\
\hline Norte & 16 & 22 & 24 & 34 & 36 & 27 & 28 & 29 & 43 & 26 & $\mathbf{2 8 5}$ \\
Centro-Oeste & 31 & 25 & 33 & 34 & 36 & 25 & 39 & 40 & 32 & 35 & $\mathbf{3 3 0}$ \\
Sul & 95 & 94 & 83 & 99 & 86 & 105 & 94 & 102 & 123 & 96 & $\mathbf{9 7 7}$ \\
Nordeste & 88 & 89 & 113 & 115 & 121 & 101 & 131 & 105 & 130 & 106 & $\mathbf{1 0 9 9}$ \\
Sudeste & 232 & 253 & 259 & 260 & 257 & 277 & 270 & 279 & 281 & 260 & $\mathbf{2 6 2 8}$ \\
\hline TOTAL & $\mathbf{4 6 2}$ & $\mathbf{4 8 3}$ & $\mathbf{5 1 2}$ & $\mathbf{5 4 2}$ & $\mathbf{5 3 6}$ & $\mathbf{5 3 5}$ & $\mathbf{5 6 2}$ & $\mathbf{5 5 5}$ & $\mathbf{6 0 9}$ & $\mathbf{5 2 3}$ & $\mathbf{5 3 1 9}$ \\
\hline
\end{tabular}

Fonte: Autoral baseado nos dados de INCA, (2020)a. 
O ano de 2017 foi o pico de notificações para todas as regiões brasileiras, 11,49\% (n=609), com exceção do CentroOeste, no qual o auge de casos foi registrado em 2016. Enquanto isso, o ano de 2009 apresentou o menor registro de óbitos, 8,68\% ( $\mathrm{n}=462)$, sendo assim para as regiões Norte, Nordeste e Sudeste. O Sul e o Centro-Oeste mostram as menores notificações nos anos de 2011 e 2010/2014 respectivamente.

Na Tabela 2, a maior quantidade de desfecho em óbitos pelo LH ocorre em homens adultos, 30,93\% (n = 1645); seguido por mulheres adultas, 23,46\% $(\mathrm{n}=1248)$; homens idosos, 20,92\% ( $\mathrm{n}=1113)$; mulheres idosas, 17,54\% $(\mathrm{n}=933)$; homens jovens, $4,41 \%(\mathrm{n}=235)$ e, por fim, as mulheres jovens, as quais apresentam o menor número de notificação para esse resultado, 2,68\% $(\mathrm{n}=143)$. Em todo território nacional, apenas um registro apresentou falha no preenchimento da faixa etária, o qual ocorreu no Centro-Oeste. Sendo assim, de todas as notificações registradas no território brasileiro, os homens representam 56,29\% $(\mathrm{n}=2994)$ das notificações, enquanto as mulheres 43,71\% $(\mathrm{n}=2324)$, o que representa uma proporção próxima de 1,3 mortes do sexo masculino para cada uma do sexo feminino.

Tabela 2: Número de óbitos pelo Linfoma de Hodgkin no Brasil, por região, de acordo com o sexo e a faixa etária entre 2009 e 2018.

\begin{tabular}{ccccc|ccccc}
\hline & \multicolumn{3}{c|}{ MASCULINO } & \multicolumn{5}{c}{ FEMININO } \\
\hline Norte & Jovens & Adultos & Idosos & Ignorado & Jovens & Adultos & Idosos & Ignorado & TOTAL \\
Centro-Oeste & 34 & 100 & 57 & 00 & 16 & 50 & 28 & 00 & $\mathbf{2 8 5}$ \\
Sul & 31 & 105 & 66 & 01 & 13 & 83 & 49 & 00 & $\mathbf{3 3 0}$ \\
Nordeste & 73 & 363 & 214 & 00 & 39 & 256 & 154 & 00 & $\mathbf{1 0 9 9}$ \\
Sudeste & 84 & 778 & 558 & 00 & 56 & 647 & 504 & 00 & $\mathbf{2 6 2 7} *$ \\
\hline TOTAL & $\mathbf{2 3 5}$ & $\mathbf{1 6 4 5}$ & $\mathbf{1 1 1 3}$ & $\mathbf{0 1}$ & $\mathbf{1 4 3}$ & $\mathbf{1 2 4 8}$ & $\mathbf{9 3 3}$ & $\mathbf{0 0}$ & $\mathbf{5 3 1 8}$ \\
\hline
\end{tabular}

*Nos dados oficiais do Atlas de Mortalidade por Câncer do INCA, a população idosa masculina do sudeste soma 558 casos de óbitos, enquanto a população idosa feminina soma 504 casos de óbitos. No entanto, eles apresentam a soma dos casos como 1063 quando deveria ser 1062, o que integraliza o número de 2628 casos na região Sudeste e assim 5319 casos no Brasil.

Fonte: Autoral baseado nos dados de INCA, (2020)a.

Ao analisar a faixa etária "jovens", entre os casos totais registrados em cada região, o Norte apresenta maior proporção de óbitos, uma vez que $\operatorname{dos} 285,17,54 \%(\mathrm{n}=50)$ pertencem a esta região. Em seguida, aparecem em ordem decrescente, o Nordeste, 10,19\% ( $\mathrm{n}=112)$; Centro-Oeste, 7,78\% ( $\mathrm{n}=26)$; Sudeste, 5,32\% ( $\mathrm{n}=140)$ e a região Sul, com apenas $5,11 \%(\mathrm{n}=50)$ das mortes nesse intervalo de idades.

Os registros na população adulta, dentro dos casos de cada região, se destacou o Centro-Oeste, 56,96\% $(\mathrm{n}=188)$, com proporção similar o Nordeste com 56,32\% ( $\mathrm{n}=619)$; Sudeste, 54,24\% ( $\mathrm{n}=1425)$; Norte, 52,63\% $(\mathrm{n}=150)$ e a região sul com $52,30 \%(\mathrm{n}=511)$.

Para os casos de morte na população idosa, a região Sul registrou a maior taxa, 42,57\% $(\mathrm{n}=416)$; em seguida o Sudeste 40,42\% ( $\mathrm{n}=1062)$; Centro-Oeste 34,84\% $(\mathrm{n}=115)$; Nordeste 33,48\% $(\mathrm{n}=368)$; e com a menor proporção a região Norte com $29,82 \%(\mathrm{n}=85)$.

Na Tabela 3, é evidenciada uma grande diferença entre os casos de morte por LH nos estados que compõe o Norte brasileiro. Enquanto o estado do Pará foi responsável por 43,13\% (n = 123) das notificações dessa região, o Acre registrou apenas 3,15\% ( $\mathrm{n}=9)$. Entre esses extremos se encontram Amazonas, 24,56\% ( $\mathrm{n}=70)$; Rondônia, 9,82\% ( $\mathrm{n}=28)$; Tocantins com 9,12\% ( $\mathrm{n}=26)$; Amapá, 5,26\% ( $\mathrm{n}=15)$ e Roraima, 4,91\% ( $\mathrm{n}=14)$. 
Tabela 3: Número de óbitos pelo Linfoma de Hodgkin na região Norte, por estado, de acordo com o sexo e a faixa etária entre 2009 e 2018.

\begin{tabular}{ccccc|cccc|c}
\hline & \multicolumn{3}{c}{ MASCULINO } & \multicolumn{5}{c}{ FEMININO } \\
\hline Acre & Jovens & Adultos & Idosos & Ignorado & Jovens & Adultos & Idosos & Ignorado & TOTAL \\
Roraima & 02 & 03 & 02 & 00 & 01 & 01 & 00 & 00 & $\mathbf{0 9}$ \\
Amapá & 02 & 07 & 00 & 00 & 00 & 04 & 02 & 00 & $\mathbf{1 5}$ \\
Tocantins & 03 & 11 & 05 & 00 & 01 & 04 & 02 & 00 & $\mathbf{2 6}$ \\
Rondônia & 03 & 10 & 08 & 00 & 01 & 02 & 04 & 00 & $\mathbf{2 8}$ \\
Amazonas & 11 & 18 & 18 & 00 & 06 & 13 & 04 & 00 & $\mathbf{7 0}$ \\
Pará & 12 & 45 & 22 & 00 & 05 & 24 & 15 & 00 & $\mathbf{1 2 3}$ \\
\hline TOTAL & $\mathbf{3 4}$ & $\mathbf{1 0 0}$ & $\mathbf{5 7}$ & $\mathbf{0 0}$ & $\mathbf{1 6}$ & $\mathbf{5 0}$ & $\mathbf{2 8}$ & $\mathbf{0 0}$ & $\mathbf{2 8 5}$ \\
\hline
\end{tabular}

Fonte: Autoral baseado nos dados de INCA, (2020)a.

Os óbitos relacionados à faixa etária na região seguem o padrão nacional, de forma crescente, jovens, $17,54 \%$ ( $\mathrm{n}=$ $50)$; idosos, $29,82 \%(\mathrm{n}=85)$ e adultos $52,63 \%(\mathrm{n}=150)$. No entanto, a fração dos casos entre os sexos se alteram de forma significa em relação à nacional, uma vez que houve 67,01\% $(\mathrm{n}=191)$ de registros para o gênero masculino e 32,99\% $(\mathrm{n}=94)$ para o feminino, resultando em uma proporção de um pouco mais de dois homens para cada uma mulher.

Na Tabela 4, no estado do Amapá, a capital, Macapá, notificou 80,00\% ( $\mathrm{n}=12$ ) das mortes por LH no estado do Amapá, enquanto o restante de 20,00\% $(\mathrm{n}=3)$ foi dividido entre três municípios: Amapá $(\mathrm{n}=1)$, Porto Grande $(\mathrm{n}=1)$ e Santana $(n=1)$.

Tabela 4: Número de óbitos pelo Linfoma de Hodgkin no estado do Amapá, por cidade, de acordo com o sexo e a faixa etária entre 2009 e 2018.

\begin{tabular}{ccccc|cccc|c}
\hline & \multicolumn{3}{c|}{ MASCULINO } & \multicolumn{5}{c}{ FEMININO } \\
\hline & Jovens & Adultos & Idosos & Ignorado & Jovens & Adultos & Idosos & Ignorado & TOTAL \\
Macapá & 02 & 05 & 00 & 00 & 00 & 03 & 02 & 00 & $\mathbf{1 2}$ \\
Amapá & 00 & 01 & 00 & 00 & 00 & 00 & 00 & 00 & $\mathbf{0 1}$ \\
Porto Grande & 00 & 01 & 00 & 00 & 00 & 00 & 00 & 00 & $\mathbf{0 1}$ \\
Santana & 00 & 00 & 00 & 00 & 00 & 01 & 00 & 00 & $\mathbf{0 1}$ \\
Demais Municípios & 00 & 00 & 00 & 00 & 00 & 00 & 00 & 00 & $\mathbf{0 0}$ \\
\hline TOTAL & $\mathbf{0 2}$ & $\mathbf{0 7}$ & $\mathbf{0 0}$ & $\mathbf{0 0}$ & $\mathbf{0 0}$ & $\mathbf{0 4}$ & $\mathbf{0 2}$ & $\mathbf{0 0}$ & $\mathbf{1 5}$ \\
\hline
\end{tabular}

Fonte: Autoral baseado nos dados de INCA, (2020)a.

No Amapá, os registros entre os idosos, 13,33\% $(\mathrm{n}=2)$ se igualam com os dos jovens, $13,33 \%(\mathrm{n}=2)$, com a peculiaridade de não haver notificações para homens idosos e mulheres jovens. Os adultos representam 73,33\% $(\mathrm{n}=11)$ das notificações.

A proporção de óbitos entre os sexos se aproxima da nacional, visto que houve 60,00\% $(\mathrm{n}=9)$ de registros para o sexo masculino e 40,00\% ( $n=6)$ para o feminino, resultando em uma fração de 1,5 homens para cada mulher com desfecho de morte. 


\section{Discussão}

Os óbitos por LH no Brasil, no recorte temporal estudado, apresentam um comportamento singular (Tabela 1), posto que entre os primeiros quatro anos houve aumento no número total e depois oscilação, muitos fatores podem estar implicados. O tempo de diagnóstico, a disponibilidade da terapêutica empregada e a qualidade da equipe médica são relevantes nisso, visto que em algumas regiões do país estes estão em constante desenvolvimento. Isso ratifica a concordância com a literatura que os óbitos por LH são menores comparados a outros tipos de câncer (Freitas, Dias, e Reis, 2020; INCA, 2020b; Monteiro, Arnaud, Monteiro, Da Costa, e Vasconcelos, 2016).

Com relação às taxas demográficas, o Sudeste apresentou maior número de óbitos, enquanto o Norte, o menor. Comparativamente são regiões distintas e longínquas, com aspectos socioeconômicos extremos. Pode-se inferir que tais demandas se dão à localização de centros especializados, levando pessoas de diferentes regiões a procurar atendimento e acompanhamento na região Sudeste tanto por motivação própria quanto por referência de serviços locais, onde se concentra um maior número de centros de hemoterapia e de oncologia. O esclarecimento, a divulgação de informações e a busca por atendimento médico de qualidade e especializado e as consequentes internações pela doença são vistas em pessoas mais jovens e corroboram ao maior número de mortes na região Sudeste e menor da região Norte brasileira (Monteiro et al., 2016).

Outro aspecto bem estabelecido é a população sudestina ser a maior do país, com mais de 89 milhões de habitantes, enquanto que a região Norte fica a frente apenas da região Centro-Oeste. Isso possui ligação íntima com o maior número de casos no sudeste. Ademais, esta região possui maior prevalência de pessoas vivendo com o vírus da imunodeficiência humana (HIV), o qual comprovadamente possui ligação íntima com o desenvolvimento de linfomas e entre eles, o linfoma de Hodgkin com risco até 30 vezes maior (Instituto Brasileiro de Geografia e Estatística [IBGE], 2020; Ministério da saúde, 2019; Nascimento, 2016).

Nessa perspectiva, verifica-se que a população com maior número de óbitos é a de adultos em todas as regiões brasileiras e em ambos os gêneros, seguido pelos idosos (Tabela 2), concordando com os dados de número de internações por LH no país que tem maior incidência no intervalo de idade entre 20 a 29 anos. Além disso, os dados são peculiares quanto à incidência na literatura, pois esta afirma que em países em desenvolvimento, o qual o Brasil se encaixa, apresenta maior incidência em crianças, entretanto, os dados mostram que as crianças possuem melhor prognóstico entre todas as idades com menos mortes (Fernandes et al., 2020; Rodrigues, Voigt, Turmina, Hata e Fiori, 2020).

Ademais, o pico bimodal de incidência no mundo e em países desenvolvidos, conforme a literatura prevalece como padrão no índice de mortes em todas as regiões (Tabela 2), sendo o primeiro entre os adultos jovens e o segundo pico nos idosos. Isso contraria a posição do país diante dos demais países em desenvolvimento e subdesenvolvidos (Brasil, 2020; Kim, Silver, Li, Neuberg, \& Mauch, 2003).

Dados levam a considerar que as causas das mortes em idosos serem maiores que em crianças são devidas não somente à idade, mas também ao estágio avançado da doença, ao tempo do diagnóstico, a pouca tolerância ao tratamento que por vezes é agressivo e também à subnotificação posto que estes podem morrer por outras causas no intercurso do adoecimento. Além disso, as regiões mais economicamente desenvolvidas do país, sudeste e sul, concentram maior número de mortes em idosos, após os adultos, em acordo com o padrão existente em países desenvolvidos. (Barros, 2007; Kim et al., 2003).

Quanto ao gênero, a literatura é divergente em suas afirmações, haja vista que há afirmações acerca de não existir grande diferença quanto à incidência entre os gêneros masculino e feminino. Por outro lado, há dados que indicam maior incidência em homens bem como análises que colocam o LH com maior predominância em mulheres entre os cânceres que mais afetam as duas populações. De fato, este estudo mostra que nos homens predominam o número de mortes em todas as idades e em todas as regiões demográficas do país (Tabela 2) e ratificando os homens adultos com maior número de óbitos. 
Este dado se repete quanto à região Norte, onde o número de óbitos masculinos é superior a duas vezes ao feminino (Tabela 3) (Fernandes et al., 2020; Freitas et al., 2020; Monteiro et al., 2016; Rodrigues e Ferreira, 2010).

O padrão estabelecido pela região Norte (Tabela 3) é concordante ao do país, no qual há mais óbitos em adultos e menos em jovens e em crianças, predominando o sexo masculino concordando com os dados incidentes de demais países em desenvolvimento também. Contudo, alguns estados mostram pequena diferença entre as faixas etárias, confirmando a baixa letalidade da doença, mas discordando da distribuição bimodal de incidência da doença (Fernandes et al., 2020; Freitas et al., 2020).

Os estados do Pará e do Amazonas destacaram-se quanto ao maior número de óbitos na região Norte. Em especial, o Pará apresentou mais mortes, isso é esperado ao saber que neste estado os linfomas são a $5^{\text {a }}$ neoplasia mais incidente. Esses dados possuem relação causal assim como o padrão registrado na região Sudeste em relação ao Brasil, pois as capitais Belém e Manaus concentram maior densidade demográfica bem como maior número de centros especializados (Monteiro, 2010; Monteiro et al., 2016)

O estado do Amapá concentra a $5^{\mathrm{a}}$ maior taxa de óbitos por LH na região Norte (Tabela 3), para isso deve-se considerar aspectos como o isolamento geográfico do estado, o baixo número de profissionais especializados, bem como um único centro de referência (Unidade de Alta Complexidade em Oncologia [UNACON]) deficitário com falta de medicações para realização de quimioterapia e a inexistência de radioterapia no estado, levando os pacientes a outros estados da federação para o tratamento necessário (Barbosa et al., 2015, INCA, 2019; Pacheco, 2016; Santiago, 2020).

Em adição a estes dados, o estado do Amapá mostra números singulares (Tabela 4) e concordantes às informações supracitadas. A capital, Macapá, concentra o maior número de óbitos, sendo estes entre adultos em ambos os sexos, isto devido aos mesmos motivos já discutidos sobre localidades com maiores taxas de morte. Demais municípios apresentaram taxa inferior, sem causa aparente, posto que o diagnóstico seja feito de maneira histológica. Resta inferir que são pacientes desassistidos pelo sistema de saúde de alta complexidade. Por outro lado, deve-se atentar que as demais faixas etárias, jovens e idosos, apresentam números baixos, fazendo com que a doença no estado não assuma aspecto bimodal, mas apenas um pico em conformidade com a faixa etária mais incidente da doença, os adultos jovens e apresentando uma baixa letalidade (Brasil, 2020; Freitas et al., 2020).

É cristalino observar que a população encontra dificuldades quanto à informação sobre a doença, uma barreira que deve ser vencida, pois esta é uma questão encontrada em doenças mais incidentes como a tuberculose. Em conformidade a isso, a subnotificação é um aspecto observado em demais doenças no estado do Amapá, essas informações são importantes de serem salientadas principalmente na população idosa (Ribeiro, Soares, Barbosa, e Almeida, 2020; Santana, Magrini, Ribeiro, Rigelli, e Araújo, 2020).

Aspectos psicológicos pessoais e familiares devem ser considerados nessas situações, desde a dificuldade de encontrar o tratamento correto e sua disponibilidade, como ocorre no estado do Amapá, e até encarar o luto diante de um diagnóstico de doença maligna e por vezes lidar com a morte (Silveira, Costa, Lohmann, e Lavall, 2020).

Diante disso, observa-se que o estudo apresentado possui como fator limitante estabelecer a precisão dos dados, pois estes dependem de análise técnica e podem até levar a subnotificações como acontece pela morte por outras causas e até mesmo o desconhecimento do diagnóstico. Contudo, as informações levantadas são de relevância epidemiológica e podem ser utilizadas para traçar planejamentos de assistência à saúde em todos os níveis de assistência no país.

\section{Considerações Finais}

Os números de desfechos em morte por LH no período de 10 anos estudados permite uma análise interessante acerca do comportamento da doença nas populações brasileira, nortista e amapaense. O Brasil possui dimensão continental e de 
desigualdade econômica e social, isso reflete nos dados de saúde pública em diversas doenças e foi identificado nos óbitos por LH. O sudeste concentra o maior número de mortes chegando a ser cerca de 10 vezes maior que a região com menor índice, a região Norte.

Cidades muito populosas, concentração de profissionais de saúde especializados, maior fluxo de informações e maior número de centros de especialidades oncológicas são fatores que concentram maiores números de óbitos por LH devido a uma migração da população doente em busca de melhor qualidade de sobrevida e de maiores chances de cura.

Dados interessantes foram observados quanto ao perfil daqueles que mais morrem, estes são homens, em idade adulta com desfecho em grandes centros. Em sequência, têm-se idosos e homens. Jovens e crianças possuem menor taxa de letalidade, que pode inferir-se por um diagnóstico precoce e pela idade apresentada ao diagnóstico, levando a um melhor prognóstico.

Por fim, é necessário que haja uma busca ativa reforçada sobre a doença, bem como a propagação de informações por meio de campanhas, a notificação correta da causa de óbito e o controle de casos novos, principalmente na população adulta, em todo o território nacional.

\section{Referências}

Barbosa, S. F. C., Costa, C. A., Ferreira, L. S. C., Almeida, D. S., Azevedo, T. C. B., Lemos, J. A. R., \& Sousa, M. S. (2015). Aspectos epidemiológicos dos casos de leucemia e linfomas em jovens e adultos atendidos em hospital de referência para câncer em Belém, Estado do Pará, Amazônia, Brasil. Revista panamazônica de saúde 6(1), 43-50. doi: 10.5123/S2176-62232015000300006.

Barros, M. H. M. (2007). Linfoma de Hodgkin na infância e adolescência: um estudo das características histológicas, clínicas, epidemiológicas e de associação com o vírus Epstein-Barr. Dissertação de mestrado, Instituto Nacional do Câncer, Rio de Janeiro, RJ, Brasil.

Brasil. (2020). Diretrizes Diagnósticas e Terapêticas do Linfoma de Hodgkin. Recuperado em 23 novembro, 2020, de http://conitec.gov.br/images/Consultas/Relatorios/2020/DDT_Linforma_de_Hodgkin_CP_11_2020.pdf.

Carbone, P. P., Kaplan, H. S., Musshoff, K., Smithers, D. W., \& Tubiana, M. (1971). Report of the Committee on Hodgkin's Disease Staging Classification. Cancer research 31, 1860-1861.

Cheson, B. D., Fisher, R. I., Barrington, S.F., Cavalli, F., Schwartz, L. H., Zucca, E., \& Lister, T. A. (2014). Recommendations for initial evaluation, staging, and response assessment of Hodgkin and non-Hodgkin lymphoma: The lugano classification. Journal of clinical oncology 32(27), 3059-3067. doi: $10.1200 / \mathrm{JCO} .2013 .54 .8800$

Duarte, F. B., Fernandes, M. G. B, Kaufmann, J., Barroso, K. S. N., Leitão, J. P. V., Araújo, B. S. G. S., Da Costa, C. M. B. E., Teles, A., Holanda, J. S., Landim, S. V., \& Pitombeira, M. H. (2016). Hodgkin's lymphoma - evaluation of patients submitted to autologous transplantation of hematopoietic cells in the hematology service of the hospital Walter Cantídio - Fortaleza, Brazil. Revista da associação médica brasileira 62(1), 34-38. http://dx.doi.org/10.1590/1806-9282.62.supp11.34.

Fernandes, A. J. S., Voigt, A. D., Turmina, L., Hata, M. M., \& Fiori, C. M. C. M. (2020). Linfoma de Hodgkin em crianças e adolescentes: Estudo clínico e epidemiológico. Revista Thêma et Scientia 10(1), 36-46.

Fernandes, J. F., Borges, A. C., Sarques, V. B., Barreto, L. P., Diniz, H. C., Dib, M. C. R. E., Carrijo, B. V., Rego, C. E. M., Sampaio, L. C. F., \& Neves, R. A. (2020). Análise epidemiológica das internações por linfoma de Hodgkin no Brasil. Hematology, transfusion and cell therapy 42(S2), S202. https://doi.org/10.1016/j.htct.2020.10.340 .

Fields, P., \& Wrench, David. (2017). Hodgkin lymphoma. Medicine 45(5), 305-310.

Freitas, F. T., Dias, J. L., \& Reis, B. C. C. (2020). Análise epidemiológica de pacientes com linfoma de hodgkin nos últimos cinco anos no estado do Rio de Janeiro. Revista de saúde 11(1), 64-66.

Gobbi, P. G., Ferreri, A. J. M., Ponzoni, M., \& Levis, A. (2013). Hodgkin lymphoma. Critical reviews in oncology/hematology 85(2), 216-237. https://dx.doi.org/10.1016/j.critrevonc.2012.07.002.

Hjalgrim, H., Askling, J., Rostgaards, K., Hamilton-Dutoit, S., Frisch, M., Zhang, J. S., Madsen, M., Rosdahl, N., Konradsen, H. B., \& Melbye, M. (2003). Characteristics of Hodgkin's lymphoma after infectious mononucleosis. The new england journal of medicine 349, 1324-1332. DOI: 10.1056/NEJMoa023141.

Instituto Brasileiro de Geografia e Estatística. (2020). Estimativas da população residente no Brasil e unidades da federação com data de referência em $1^{o}$ de julho de 2020.https://ftp.ibge.gov.br/Estimativas_de_Populacao/Estimativas_2020/estimativa_dou_2020.pdf

Instituto Nacional do Câncer. (2020a). Atlas de mortalidade por câncer. https://www.inca.gov.br/aplicativos/atlas-de-mortalidade-por-cancer.

Instituto Nacional do Câncer. (2020b). Estimativas 2020. https://www.inca.gov.br/estimativa/estado-capital/brasil.

Instituto Nacional do Câncer. (2019). Onde tratar pelo SUS. https://www.inca.gov.br/onde-tratar-pelo-sus 
Kim, H. K., Silver, B., Li, S., Neuberg, D., \& Mauch, P. (2003). Hodgkin's disease in elderly patients (>60): clinical outcome and treatment strategies. International journal of radiology, biology, physics 56(2), 556-560. doi:10.1016/S0360-3016(02)04596-0.

Küppers, R., Engert, A., \& Hansmann, M. (2012). Hodgkin lymphoma. The journal of clinical investigations 122(10), 3439-3447. doi:10.1172/JCI61245.

Ministério da saúde. (2019). HIV/Aids 2019. http://www.aids.gov.br/pt-br/pub/2019/boletim-epidemiologico-de-hivaids-2019.

Monteiro, T. A. F., Arnaud, M. V. C., Monteiro, J. L. F., Da Costa, M. R. M., \& Vasconcelos, P. F. C. (2016). Linfoma de Hodgkin: aspectos epidemiológicos e subtipos diagnosticados em um hospital de referência no Estado do Pará, Brasil. Revista pan-amazônica de saúde 7(1), 27-31. doi: 10.5123/S2176622320016000100003 .

Monteiro, T. A. F. (2010). Linfoma de Hodgkin na infância e adolescência: um estudo das características histológicas, clínicas, epidemio - lógicas e de associação com o vírus Epstein-Barr. Dissertação de mestrado, Universidade Federal do Pará, Belém, PA, Brasil.

Nascimento, J. S. (2016). Linfomas relacionados ao HIV em adultos atendidos na rede pública de Recife-PE. Dissertação de mestrado, Universidade Federal de Pernambuco, Recife, PE, Brasil.

Pacheco, J. (2016). Familiares apontam falta de remédios e materiais para tratamento de câncer. http://g1.globo.com/ap/amapa/noticia/2016/09/familiaresapontam-falta-de-remedios-e-materiais-para-tratamento-de-cancer.html.

Ribeiro, L. A., Soares, N. J. A., Barbosa, R. E. A., \& Almeida, E. M. R. (2020). Tuberculose pleural em paciente sob situação de risco: relato de caso. Revista científica multidisciplinar núcleo do conhecimento 10(4), 121-142. 10.32749/nucleodoconhecimento.com.br/saude/tuberculose-pleural.

Rodrigues, J. S. M. \& Ferreira, N. M. L. A. (2010). Caracterização do Perfil Epidemiológico do Câncer em uma Cidade do Interior Paulista: Conhecer para Intervir. Revista brasileira de cancerologia 56(4), 431-441.

Santiago, A. (2020). Sem radioterapia no Amapá, pacientes com câncer lutam para sobreviver. https://www.uol.com.br/vivabem/noticias/redacao/2020/08/05/na-pandemia-pacientes-com-cancer-lutam-para-viver-onde-falta-tratamento.htm.

Santana, J. L. A., Magrini, A. T., Ribeiro, L. A., Rigelli, R. H., \& Araújo, M. H. M. (2020). Adesão ao tratamento da tuberculose no Amapá: um quinquênio de análise epidemiológica. Revista científica multidisciplinar núcleo do conhecimento 10(17), 69-87. 10.32749/nucleodoconhecimento.com.br/saude/tratamentoda-tuberculose.

Santos, M. A. O., \& Lima, M. M. (2017). CD20 role in pathophisiology of Hodgkin’s disease. Revista da Associação Médica Brasileira 63(9), 810-813. http://dx.doi.org/10.1590/1806-9282.63.09.810.

Silveira, P. J. da, Costa, A. E. K. da, Lohmann, P. M., \& Lavall, E. (2020). Revisão integrativa: cuidados paliativos em pacientes oncológicos. Research, Society and Development, 9(2), e144922136. https://doi.org/10.33448/rsd-v9i2.2136.

Storck, K., Brandstetter, M., Keller, U., \& Knopf, A. (2019). Clinical presentation and characteristics of lymphoma in the he ad and neck region. Head and face medicine 15(1). https://doi.org/10.1186/s13005-018-0186-0. 